\title{
Schwann cell proliferation and differentiation that is induced by ferulic acid through MEK1/ERK1/2 signalling promotes peripheral nerve remyelination following crush injury in rats
}

\author{
XIAOYAN ZHU ${ }^{1}, \mathrm{KUN} \mathrm{LI}^{1}, \mathrm{XIN} \mathrm{GUO}^{1}, \mathrm{JIAN} \mathrm{WANG}^{2}$ and YANG XIANG ${ }^{2}$ \\ ${ }^{1}$ Center of Laboratory Medicine; ${ }^{2}$ Department of Neurology, Chengdu Military General Hospital, \\ Chengdu, Sichuan 610083, P.R. China
}

Received March 12, 2015; Accepted May 10, 2016

DOI: $10.3892 /$ etm.2016.3525

\begin{abstract}
Schwann cell proliferation and differentiation is critical for the remyelination of injured peripheral nerves. Ferulic acid (FA) is a widely used antioxidant agent with neuroprotective properties. However, the potentially beneficial effects of FA on Schwann cells are unknown. Therefore, the present study was designed to examine the effects of FA on Schwann cell proliferation and differentiation. By using the cultured primary Schwann cells and proliferation assay, the results identified that FA was capable of increasing Schwann cell proliferation and expression of myelin-associated glycoprotein (MAG) and myelin basic protein (MBP) in vitro. It was also observed that the beneficial effect of FA treatment on Schwann cells was mainly dependent on the activation of MEK1/ERK1/2 signalling. Furthermore, FA was intraperitoneally administered to rats with sciatic nerve crush injury, and the results revealed an increase in Schwann cell proliferation and differentiation, while the MAG and MBP expression levels in sciatic nerves were markedly upregulated following FA administration. In conclusion, the current results demonstrate that Schwann cell proliferation and differentiation is induced by FA through MEK1/ERK1/2 signalling and that FA may accelerate injured peripheral nerve remyelination.
\end{abstract}

\section{Introduction}

Schwann cells are specialised glial cells and myelin-forming cells in the peripheral nervous system, which serve a critical role in nerve remyelination after injuries (1). Following peripheral nerve damage, immature Schwann cells differentiate into a myelinating phenotype secreting myelin proteins,

Correspondence to: Dr Yang Xiang, Department of Neurology, Chengdu Military General Hospital, 270 Rongdu Avenue, Tianhui Road, Chengdu, Sichuan 610083, P.R. China

E-mail: xiangyang0440@163.com

Key words: ferulic acid, Schwann cells, proliferation, myelination, peripheral nerve injury including myelin-associated glycoprotein (MAG) and myelin basic protein (MBP), and provide a growth-permissive environment for nerve regeneration (2). It has been reported that the seeding of Schwann cells significantly promotes nerve regeneration following lesion development (3), while damage to Schwann cells induces delay of nerve growth (4). Therefore, differentiated Schwann cells with myelinating properties are required following nerve injury. In addition, the use of bioactive substances, such as fingolimod and methylcobalamin, that are capable of promoting Schwann cell proliferation and improving their myelinating properties is an important strategy for improving nerve regeneration $(5,6)$.

Ferulic acid (FA) is a phenolic compound isolated from several plants, such as Ferula assa-foetida L. The molecular structure of FA is shown in Fig. 1. FA has potent anti-oxidant and anti-inflammatory properties, and exhibits a wide range of therapeutic effects, including anticancer, antidiabetic and cardioprotective activities (7). Previous studies have indicated that FA also exerts potent neuroprotective effects, such as protecting cultured neurons against glutamate neurotoxicity (8), preserving neuronal cells against cerebral ischaemia (9) and inducing proliferation of neuronal progenitor cells (10). However, the majority of current studies concerning FA have focused on neuroprotection of neurons, while little is known about the effects of FA on Schwann cells and the signalling pathway by which FA brings about these effects.

Nerve crush injury is a well-established animal model used to investigate the impact of pharmacological treatments for nerve regeneration (11). Different chemical drugs have been investigated to improve the remyelination of the peripheral nerve in animal experiments, such as clozapine, olanzapine and quetiapine (12). However, functional recovery subsequent to peripheral injury is often poor, and severe anatomical and functional disorders are observed. Currently, naturally occurring components have gained emerging interest for their potentially beneficial use in neurodegeneration, and thus it is critical to develop pharmacological approaches that may assist in the promotion of remyelination in the regrowth of nerves.

The aims of the present study were to evaluate the impact of FA on the cell proliferation and differentiation of Schwann cells in vitro and in vivo, and to determine the effect of FA on nerve remyelination in a rat model of peripheral nerve crush 
injury. The current study provided evidence that FA significantly promoted the proliferation and expression of MAG and MBP in cultured Schwann cells through MEK1/ERK1/2 signalling transduction. The study also identified that systematic FA administration effectively promoted sciatic nerve remyelination in the crushed nerves through increasing Schwann cell proliferation and differentiation. Therefore, the present study revealed the effects of FA on Schwann cell proliferation and differentiation, and suggested a potential role for FA in the therapy of peripheral nerve injuries.

\section{Materials and methods}

Animals. Adult Sprague Dawley (SD) rats weighing 250-275 g, aged 6 months ( $\mathrm{n}=5$ per group), were provided by the Experimental Animal Center at the Chengdu Military General Hospital (Chengdu, China) and maintained with a $12 \mathrm{~h}$ light/dark cycle and ad libitum access to food and water. All procedures were performed in accordance with the Guidelines for the Care and Use of Laboratory Animals (National Institutes of Health, Bethesda, MD, USA) and were approved by the Administration Committee of Experimental Animals of Sichuan Province, China. All efforts were made to minimise the number of animals used and their suffering during the experiments.

In vitro isolation and culture of primary Schwann cells. Primary cultures of Schwann cells were obtained from the sciatic nerves of 3-day-old SD rats using the method previously described by Fei et al (13). Schwann cells were then maintained in complete Dulbecco's modified Eagle's medium (DMEM) at $37^{\circ} \mathrm{C}$ and $5 \% \mathrm{CO}_{2}$. The purity and viability of cultured primary rat Schwann cells were determined by flow cytometry with FITC-anti-S100 antibody (1:1,000; ab76749; Abcam, Cambridge, MA, USA) for Schwann cells and 7-aminoactinomycin D (BioLegend, San Diego, CA, USA) for cell viability.

Schwann cell proliferation assay. In Schwann cells in vitro proliferation assays, cells were cultured $\left(1 \times 10^{6}\right.$ cells $/ \mathrm{ml}$ for $\left.48 \mathrm{~h}\right)$ in $5 \%$ fetal bovine serum in triplicate in 96-well flat-bottom microtiter plates and treated with FA (Sigma-Aldrich, St. Louis, MO, USA). Cell proliferation was determined by cell counting kit-8 (CCK-8) assay (Dojindo Molecular Technologies, Inc., Kumamoto, Japan), as previously described (14). In ${ }^{3} \mathrm{H}$-methyl-thymidine proliferation experiments, cells were pulsed with $0.25 \mu \mathrm{Ci}$ ${ }^{3} \mathrm{H}$-methyl-thymidine (Shenggong Trade Co., Shanghai, China) at the last $16 \mathrm{~h}$ of cell culture and cell proliferation was expressed as the average counts per minute of ${ }^{3} \mathrm{H}$-thymidine uptake, as previously described (15).

Reverse transcription-quantitative polymerase chain reaction (RT-qPCR) analysis. Total RNA of Schwann cells and sciatic nerves was isolated using TRIzol LS reagent (Invitrogen; Thermo Fisher Scientific, Inc., Waltham, MA, USA) and reverse transcribed into cDNA using NCode $^{\mathrm{TM}}$ miRNA First-Strand cDNA Synthesis kit (Invitrogen; Thermo Fisher Scientific, Inc.). The resulting cDNA was used to quantitatively measure the expression of genes using Power SYBR ${ }^{\circledR}$

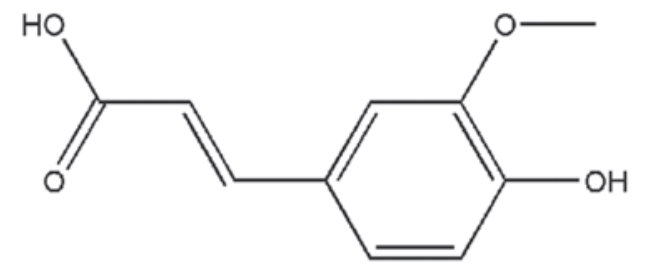

Figure 1. Molecular structure of ferulic acid.

Green Master Mix (Invitrogen; Thermo Fisher Scientific, Inc.). Primers (Wuhanboshide, China) used to measure gene expression levels were as follows: GAPDH sense, GACATG CCGCCTGGAGAAAC, and antisense, AGCCCAGGATGC CCTTTAGT; MAG sense, ACAGCGTCCTGGACATCATCA ACA, and antisense, ATGCAGCTGACCTCTACTTCCGTT; MBP sense, TTGACTCCATCGGGCGCTTCTTTA, and antisense, GCTGTGCCACATGTA CAAGGACTCA.

Western blotting. Similarly to the cell culturing in the proliferation assay, the Schwann cells were treated with FA in the absence or presence of PD98059 (Sigma-Aldrich). Schwann cells and sciatic nerves were lysed in ice-cold radioimmunoprecipitation assay buffer (Shenggong Trade Co.). The lysed cells were centrifuged $\left(12,000 \mathrm{x} \mathrm{g}, 25 \mathrm{~min}, 4^{\circ} \mathrm{C}\right)$ and the supernatants were collected. A standard western blotting protocol was used, as previously described (16). Next, the samples were incubated with primary antibodies at $4^{\circ} \mathrm{C}$ overnight, including MAG (1:500; sc-15324), MBP (1:500; sc-809), MEK1 (1:1,000; sc-219), ERK1/2 (1:1,000; sc-292838), phosphorylated (p)-MEK1 (1:500; sc-293106), p-ERK1/2 (1:500; sc-101760), which were purchased from Santa Cruz Biotechnology Inc. (Santa Cruz, CA, USA), as well as GAPDH (1:500; AB-P-R 001), from Goodhere Biotechnology Co., Ltd. (Hangzhou, China). Subsequently, the samples were incubated with the horseradish peroxidase-conjugated goat anti-rabbit IgG secondary antibody (1:500; A0208), obtained from Beyotime Institute of Biotechnology (Haimen, China). The blots were then detected by chemiluminescence using enhanced chemiluminescence reagent (BeyoECL Plus; Beyotime Institute of Biotechnology). Images of the blots were captured by Carestream Image Station 4000MM (Carestream Health Inc., Woodbridge, CT, USA), and the band density was detected by Quantity One (version 4.6.2; Bio-Rad Laboratories, Inc., Hercules, CA, USA).

Surgical procedure and treatment. Crush injury of rat sciatic nerves was performed according to the method previously described by Varejão et al (17), with minor modifications. Briefly, rats were anaesthetised, and the right sciatic nerve was carefully exposed through the gluteal-splitting approach and then crushed with a haemostatic clamp at a standardised force at moderate level for $30 \mathrm{sec}$. Animals in the treatment group $(\mathrm{n}=5)$ received FA $(50 \mathrm{mg} / \mathrm{kg}$, intraperitoneal injection) daily for 7 days after the injury, while the control group rats $(n=5)$ received the same amount of phosphate-buffered saline (PBS) injection at the same time periods. At 2 or 4 weeks after the beginning of the experiments, rats were euthanised and the right sciatic nerve segment distal to the lesion was removed 
A

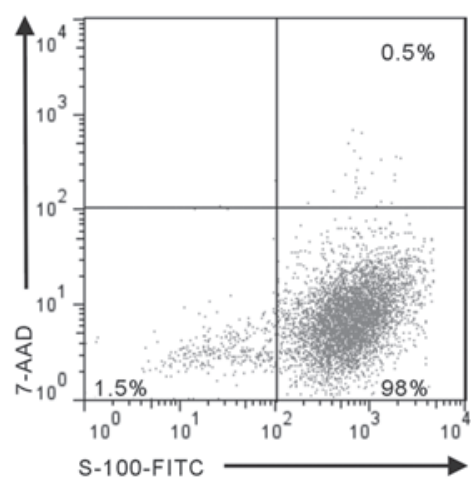

B

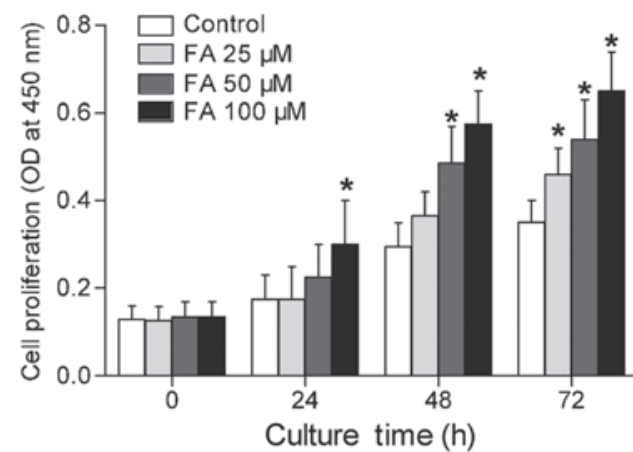

C

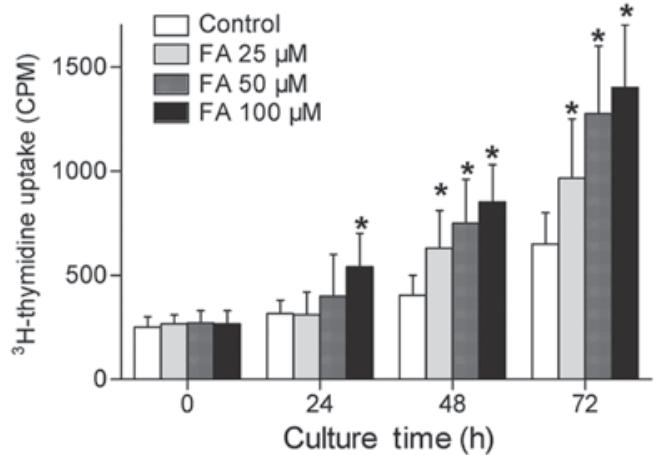

Figure 2. FA increased cell proliferation of primary Schwann cells in vitro. Schwann cells were treated with FA $(0,25,50$ and $100 \mu \mathrm{M})$ and cultured for 0, 24, 48 or $72 \mathrm{~h}$ in Dulbecco's modified Eagle's medium containing 5\% fetal bovine serum. (A) Flow cytometric identification of the purity and viability of cultured primary Schwann cells (S-100 positive for Schwann cells and 7-AAD negative for live cells). (B) Cell proliferation determined by CCK-8 assay, with the results shown as the OD at $450 \mathrm{~nm}$. (C) Cell proliferation determined by ${ }^{3} \mathrm{H}$-thymidine uptake, with the results shown as CPM. Data in the graphs are expressed as the mean \pm standard error of three separate experiments. ${ }^{*} \mathrm{P}<0.05$, vs. control. FA, ferulic acid; 7-AAD, 7-aminoactinomycin D; CCK-8, cell counting kit-8; OD, optical density; CPM, counts per minute.

and stored. Schwann cells in sciatic nerves were harvested using a rat Schwann cell isolation kit from Miltenyi Biotec GmbH (Bergisch Gladbach, Germany). The cell proliferation of isolated Schwann cells was determined by flow cytometry with a proliferation marker PE-conjugated anti-Ki-67 antibody from eBioscience Inc. (1:200; cat. no. 12-5698; San Diego, CA, USA).

Evaluation and statistical analysis. The data are expressed as the mean \pm standard error of the mean. The Mann-Whitney U test was performed to determine statistically significant differences between FA-treated and non-treated groups. GraphPad Prism version 5.0 software (GraphPad Software, Inc., La Jolla, CA, USA) was used to perform statistical analysis. Statistically significant differences were determined at $\mathrm{P}<0.05$.

\section{Results}

FA increases primary Schwann cell proliferation in vitro. Primary rat Schwann cells were successfully isolated and cultured, as demonstrated by flow cytometric analysis (Fig. 2A). In order to study the influence of FA on Schwann cells proliferation in vitro, Schwann cells were treated with various concentrations of FA $(0,25,50$ and $100 \mu \mathrm{M})$ and cultured in 5\% serum DMEM for 0, 24, 48 or $72 \mathrm{~h}$. As shown in Fig. 2B, Schwann cell viability was greatly increased following FA treatment compared with that in the control cells, as demonstrated by CCK-8 assay $(\mathrm{P}<0.05)$. Subsequently, the radioactive ${ }^{3} \mathrm{H}$-thymidine incorporation assessment was applied in order to precisely determine the effects of FA on Schwann cell proliferation. The results demonstrated that FA significantly induced Schwann cell proliferation in a dose-dependent manner (Fig. 2C). These results confirmed that FA is involved in the induction of a cell proliferative reaction of Schwann cells.

$F A$ increases the $M R N A$ and protein levels of MAG and MBP expressed by Schwann cells. MAG and MBP are important myelin proteins in peripheral nerves that are expressed by Schwann cells (18). The present study detected the MAG and MBP expression levels of cultured Schwann cells following treatment with FA by RT-qPCR and western blotting. As shown in Fig. 3, Schwann cells directly subjected to FA treatment showed an increase in their MAG and MBP mRNA expression levels (Fig. 3A and B). Furthermore, Schwann cells were subjected to FA at a therapeutic concentration $(50 \mu \mathrm{M})$ for different time periods, and the results demonstrated an upregulation of MAG and MBP mRNA expression levels subsequent to FA exposure for 24, 48 and $72 \mathrm{~h}$ (Fig. 3C and D). In accordance with the RT-qPCR results, the western blotting results also demonstrated that the protein expression levels of MAG and MBP in Schwann cells were directly promoted by FA in a concentration-dependent (Fig. 4A) and time-dependent (Fig. 4B) manner. Therefore, these data revealed that FA, beside its ability to promote Schwann cell proliferation, was also capable of inducing Schwann cell differentiation in vitro. 
A

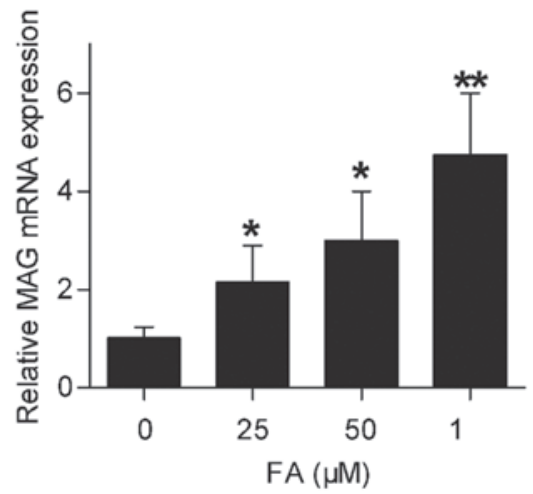

C

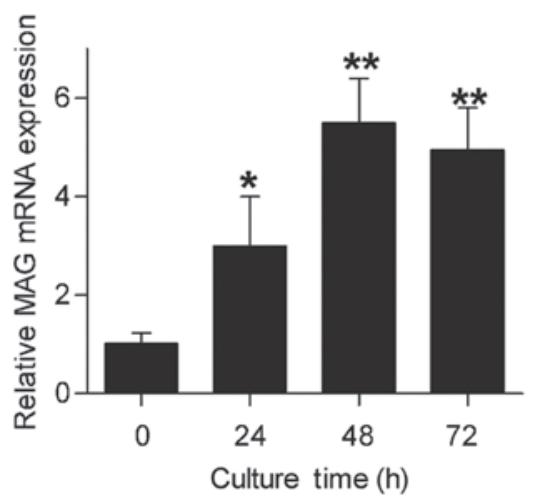

B

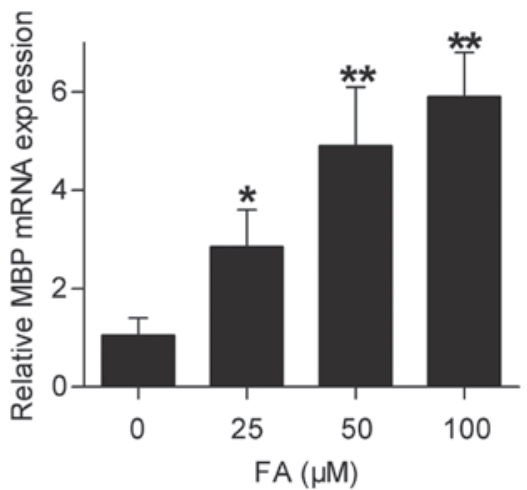

D

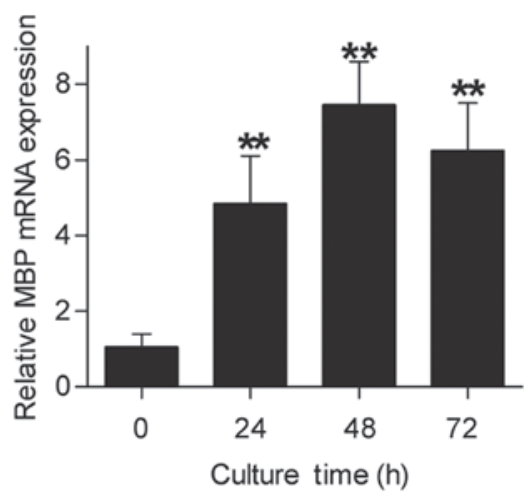

Figure 3. mRNA levels of MAG and MBP expressed by Schwann cells were upregulated by FA treatment, as determined using reverse transcription-quantitative polymerase chain reaction. (A) MAG and (B) MBP mRNA expression levels in Schwann cells treated with 0,25, 50 or $100 \mu \mathrm{M}$ FA for $24 \mathrm{~h}$. (C) MAG and (D) MBP mRNA expression levels in Schwann cells treated with $50 \mu \mathrm{M}$ FA for 0, 24, 48 or $72 \mathrm{~h}$. $\beta$-actin was used as an internal control. Data are shown as the mean \pm standard error of three separate experiments, ${ }^{*} \mathrm{P}<0.05$ and ${ }^{* * *} \mathrm{P}<0.01$, vs. $0 \mu \mathrm{M}$ FA or $0 \mathrm{~h}$ incubation. MAG, myelin-associated glycoprotein; MBP, myelin basic protein; FA, ferulic acid.

A

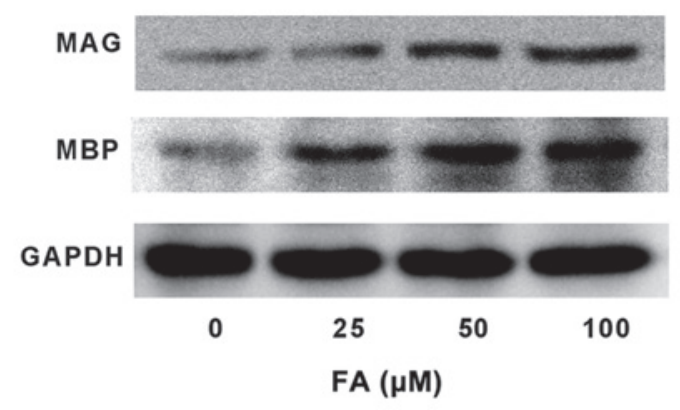

B

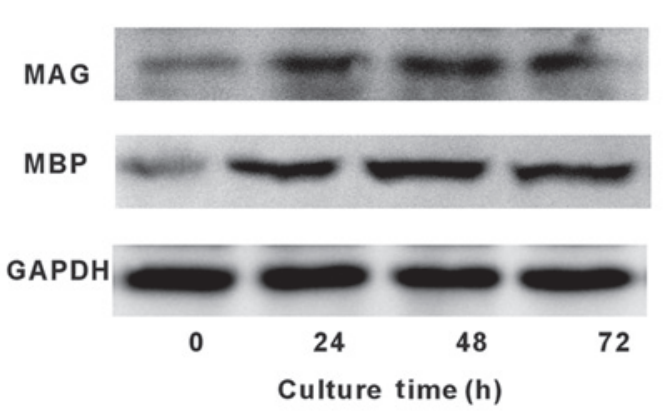

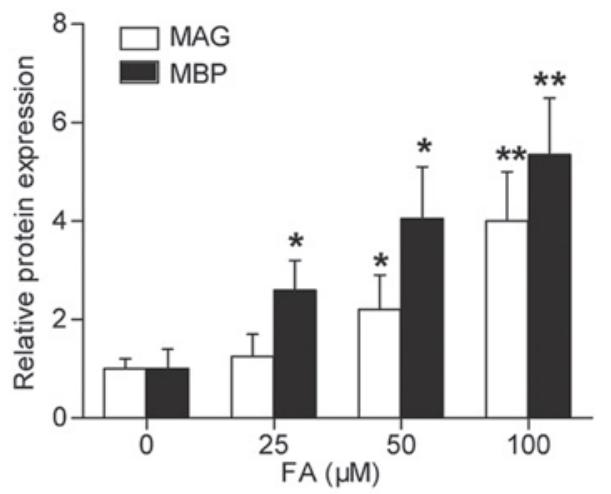

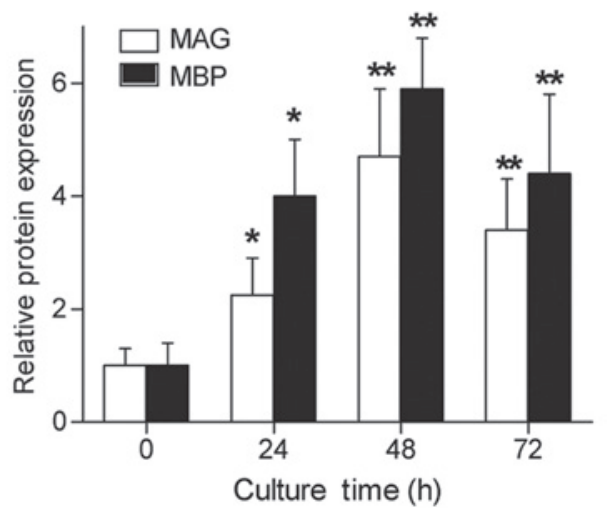

Figure 4. Protein expression levels of MAG and MBP in Schwann cells is upregulated by FA, as determined by western blotting. (A) Western blot analysis of MAG and MBP protein expression levels in Schwann cells treated with 0, 25, 50 or $100 \mu \mathrm{M}$ FA for $24 \mathrm{~h}$. (B) Western blot analysis of MAG and MBP protein expression levels in Schwann cells treated with $50 \mu \mathrm{M}$ FA for $0,24,48$ or $72 \mathrm{~h}$. GAPDH was used as an internal control. Data are shown as the mean \pm standard error of three separate experiments. ${ }^{*} \mathrm{P}<0.05$ and ${ }^{* * *} \mathrm{P}<0.01$ vs. $0 \mu \mathrm{M}$ FA or $0 \mathrm{~h}$ incubation. MAG, myelin-associated glycoprotein; MBP, myelin basic protein; FA, ferulic acid. 
A

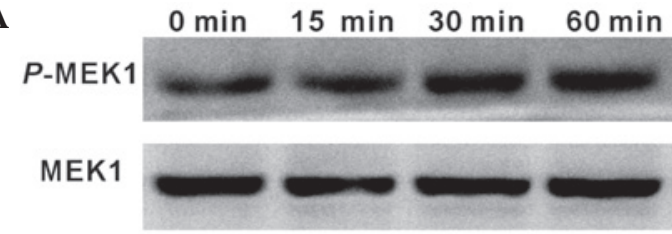

C

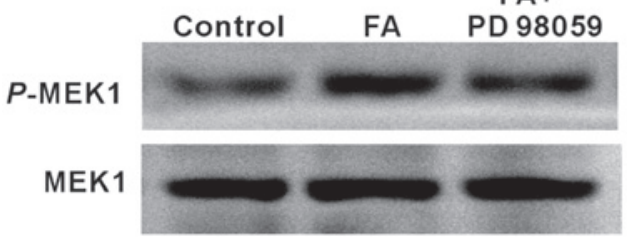

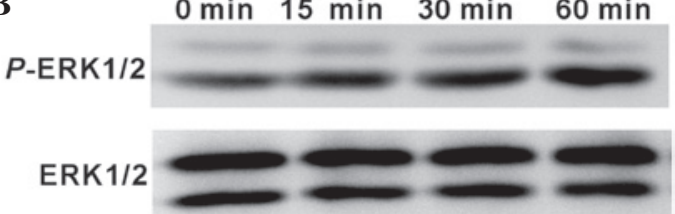

D

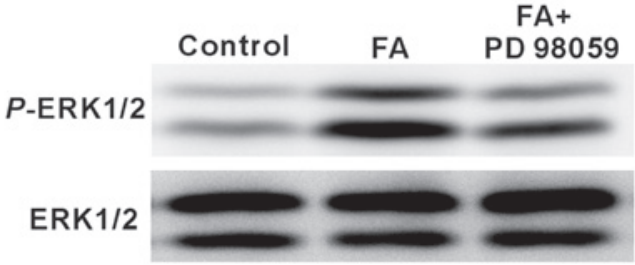

Figure 5. MEK1/ERK1/2 signalling was activated by FA in Schwann cells treated with $100 \mu \mathrm{M}$ FA with or without $20 \mu \mathrm{M}$ PD98059, a chemical inhibitor of MEK1, for indicated times. Western blots of (A) p-MEK1 and (B) p-ERK1/2 protein levels in Schwann cells treated by FA for 0, 15, 30 and 60 min are shown. However, the (C) p-MEK1 and (D) p-ERK1/2 protein levels in Schwann cells induced by FA for 60 min were downregulated in the presence of PD98059. GAPDH was used as an internal control. FA, ferulic acid.
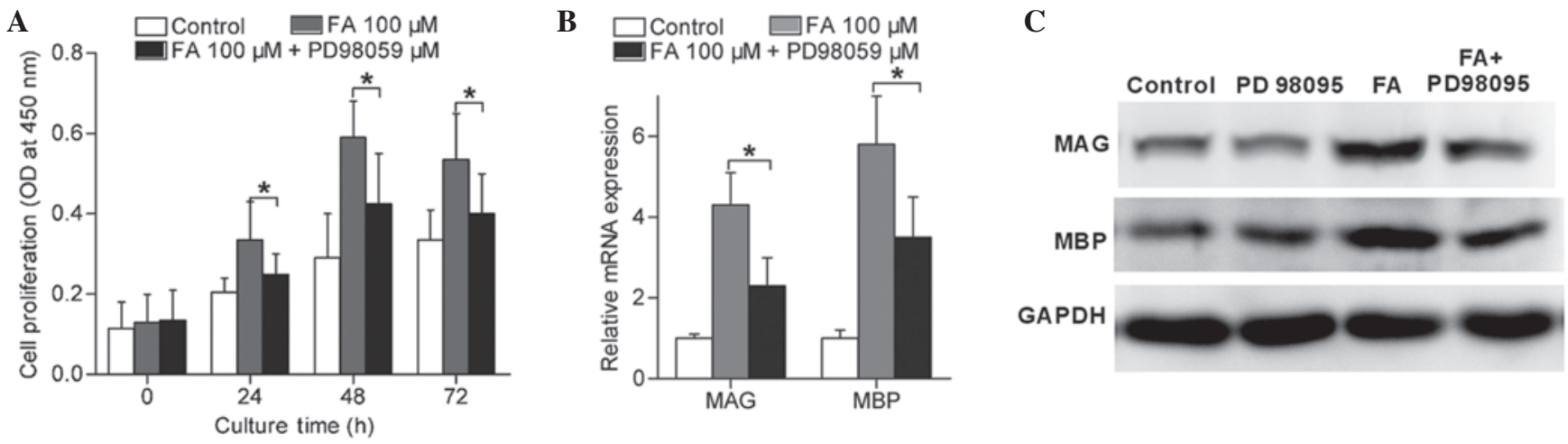

Figure 6. Inhibition of MEK1/ERK1/2 using PD98059 reduced the Schwann cell proliferation and differentiation that was induced by FA. Schwann cells were treated by $100 \mu \mathrm{M}$ FA with or without $20 \mu \mathrm{M}$ PD98059 for the indicated times. (A) Cell proliferation of Schwann cells treated by FA with or without PD98059 for 0, 24, 48 and $72 \mathrm{~h}$ was determined by CCK-8 assay. (B) MAG and MBP mRNA expressed by Schwann cells treated by FA with or without PD98059 for 24 h. (C) Protein expression levels of MAG and MBP expressed by Schwann cells treated by FA with or without PD98059 for 24 h. Data in the graphs are shown as the mean \pm standard error of three separate experiments. ${ }^{*} \mathrm{P}<0.05$. MAG, myelin-associated glycoprotein; MBP, myelin basic protein; FA, ferulic acid.

FA induces Schwann cell proliferation and differentiation through MEK1/ERK1/2 signalling. The critical role of ERK signalling pathway in Schwann cells has been previously demonstrated (19). Therefore, the present study attempted to determine whether MEK1/ERK1/2 is also activated in Schwann cells by FA. Schwann cells treated with $100 \mu \mathrm{M}$ FA (as a result of previous results shown in Fig. 2B) were harvested and analysed by western blotting. As shown in Fig. 5A and B, p-MEK1 and $\mathrm{p}$-ERK1/2 expression was induced following incubation with FA for 15, 30 and 60 min. Furthermore, when co-cultured with $20 \mu \mathrm{M}$ PD98059, a chemical inhibitor of MEK1, p-MEK1 and p-ERK1/2 expression levels in Schwann cells induced by FA were significantly inhibited (Fig. 5C and D). These results indicated that MEK1/ERK1/2 signalling in Schwann cells can be activated by FA treatment.

Furthermore, in order to determine the role of MEK1/ERK1/2 activation in FA-induced Schwann cell differentiation and proliferation, cells were incubated with $100 \mu \mathrm{M}$ FA in the presence of $20 \mu \mathrm{M}$ PD98059. The results indicated that the FA-induced Schwann cell proliferation (Fig. 6A) and expression of MAG and MBP (Fig. 6B and C) were significantly reduced in the presence of PD98059 $(\mathrm{P}<0.05)$. These findings suggested that FA induced Schwann cell proliferation and differentiation mainly through the MEK1/ERK1/2 signalling pathway.

FA treatment promoted rat sciatic nerve myelination following crush injury. Since proliferation and differentiation of Schwann cells was induced by FA in vitro, it was suggested that FA may exert similar nerve remyelination effects in vivo. Rats with sciatic nerve crush were intraperitoneally treated with FA or PBS daily for a total of 7 days. The results demonstrates that, at 4 weeks post-injury, Schwann cells isolated from FA-treated nerves showed 1-fold increase in MAG expression and 2-fold increase in MBP expression when compared with the expression levels in the PBS group $(\mathrm{P}<0.05$; Fig. 7A and B). Furthermore, Ki-67, a cell proliferation marker was used to determine the proliferation of sciatic nerve-derived Schwann cells. As shown in Fig. 7C, $35 \%$ of Schwann cells from PBS-treated sciatic nerves were Ki-67-positive, whereas this proportion was increased to $53 \%$ in FA-treated sciatic nerves, indicating that FA also 
A

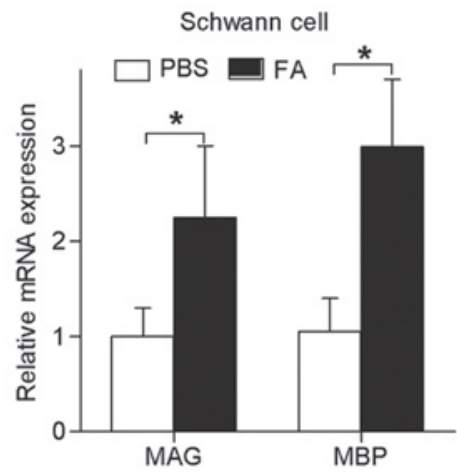

B

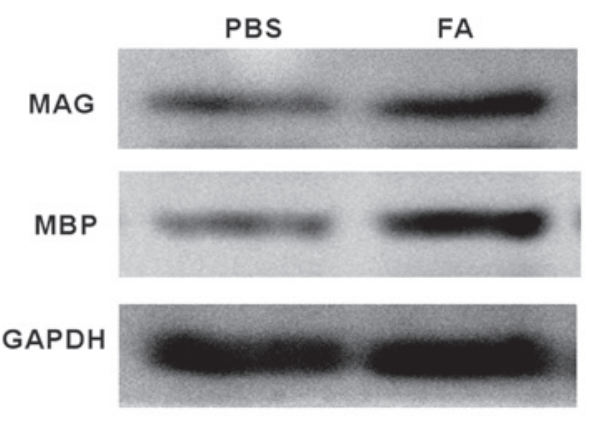

C

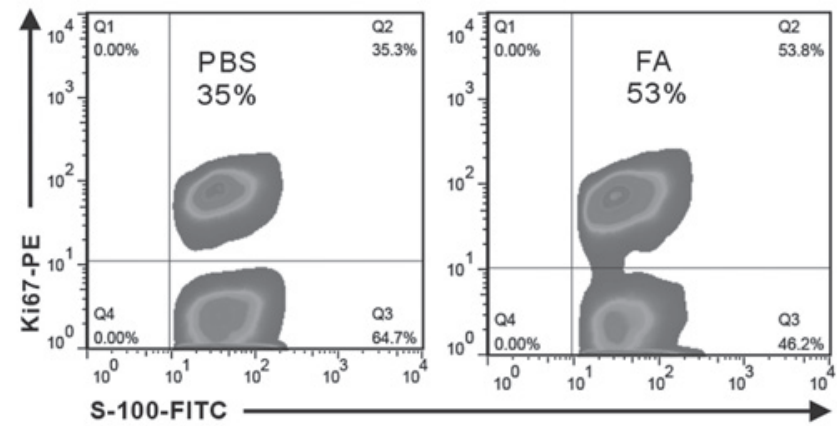

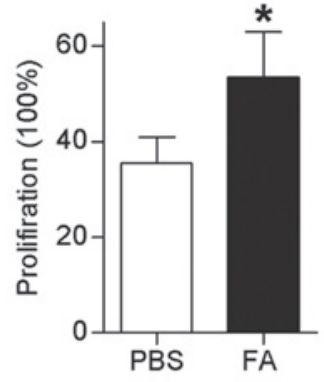

D

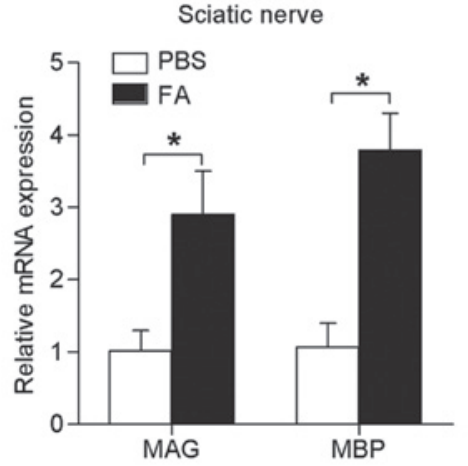

$\mathbf{E}$

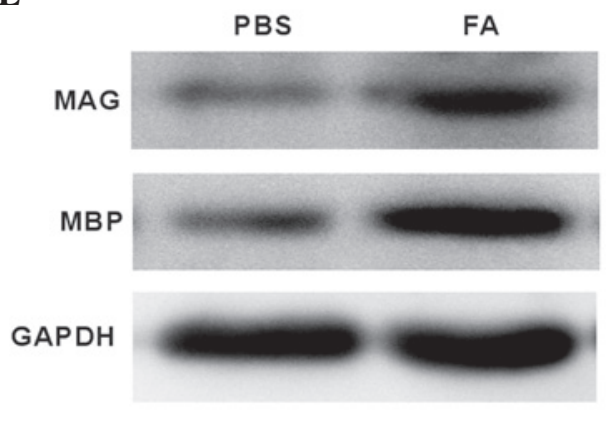

Figure 7. FA treatment promoted rat sciatic nerve myelination following crush injury. Treatment with FA ( $50 \mathrm{mg} / \mathrm{kg} / \mathrm{day}$ for 7 days; $\mathrm{n}=6)$ or PBS of the same volume $(n=6)$ was intraperitoneally injected to rats subsequent to crush injury. At 4 weeks after injury, the rats were sacrificed to extract the sciatic nerves and isolate Schwann cells for analysis. (A) mRNA and (B) protein expression levels of MAG and MBP in Schwann cells were significantly upregulated following FA treatment. (C) Ki-67-positive Schwann cells in sciatic nerves, as observed by flow cytometry. (D) mRNA and (E) protein expression levels of MAG and MBP in the sciatic nerve were significantly upregulated following FA treatment. Data in the graphs are shown as the mean \pm standard error of three separate experiments. ${ }^{*} \mathrm{P}<0.05$. MAG, myelin-associated glycoprotein; MBP, myelin basic protein; FA, ferulic acid.

exerts cell proliferation effects on Schwann cells in vivo. The expression levels of MAG and MBP in the sciatic nerve was also detected 4 weeks after crush injury. Compared with the control, FA treatment induced a 3-fold increase of MAG and 4-fold increase of MBP expression in sciatic nerves $(\mathrm{P}<0.05$; Fig. 7D and E).

\section{Discussion}

The direct function of FA on the biological properties of Schwann cells remains largely unknown. Therefore, the present study investigated the proliferative response of Schwann cells subsequent to FA exposure. In vitro assays demonstrated that FA directly induced an increase in Schwann cell proliferation, which has not been previously reported, to the best of our knowledge.
While the proliferation of Schwann cells can be induced by FA, there are currently few data regarding the effect of FA on Schwann cell differentiation. MAG has been demonstrated to serve a major role in the formation of myelin, promoting myelinogenesis and the maintenance of myelin stability through axo-glial contact in the peripheral nervous system (20). However, during the in vitro culture of Schwann cells, the biological properties of Schwann cells have been observed to significantly decline with time, limiting their application in the construction of artificial nerve grafts (21). The results of the current study revealed a marked increase in MAG and MBP expression levels in FA-treated Schwann cells, suggesting that FA may maintain and facilitate differentiation properties of cultured Schwann cells.

The present study also revealed that activation of the MEK1/ERK1/2 signalling pathway is responsible for the 
effects exerted by FA on Schwann cells. A recent study suggested a role for ERK signalling in promoting myelination due to the finding that increased ERK activity induces hypermyelination (22). Furthermore, conditional ERK knockout results have shown that ERK signalling is required for differentiation and myelination of Schwann cells (23). In the present study, western blot analysis demonstrated that the protein levels of phosphorylated MEK1/ERK1/2 in Schwann cells were evidently increased following FA treatment. In addition, PD98059, an inhibitor of MEK1, was able to suppress the activation of MEK1/ERK1/2 signalling evoked by FA treatment. Further results demonstrated that, when the MEK1/ERK1/2 transduction pathway was blocked with PD98059, the Schwann cell proliferation and differentiation induced by FA was significantly declined. Therefore, the present results are consistent with the findings of previous studies $(22,23)$, and these data suggest that activation of MEK1/ERK1/2 signalling is responsible for FA-induced cell proliferation and differentiation of Schwann cells.

In conclusion, the present study demonstrated that FA treatment was able to promote cell proliferation and expression of MAG and MBP in Schwann cells through the activation of MEK1/ERK1/2 signalling. In addition, FA was able to accelerate the remyelination of peripheral injured nerves by increasing the proliferating numbers of Schwann cells and promoting myelin secretion. These findings provide novel information on the direct effects of FA on Schwann cells and assist in developing further understanding of the neuroprotective effects produced by FA.

\section{References}

1. Hung HA, Sun G, Keles S and Svaren J: Dynamic regulation of Schwann cell enhancers after peripheral nerve injury. J Biol Chem 290: 6937-6950, 2015.

2. Chew SY, Mi R, Hoke A and Leong KW: The effect of the alignment of electrospun fibrous scaffolds on Schwann cell maturation. Biomaterials 29: 653-661, 2008.

3. Ishikawa N, Suzuki Y, Dezawa M, Kataoka K, Ohta M, Cho H and Ide C: Peripheral nerve regeneration by transplantation of BMSC-derived Schwann cells as chitosan gel sponge scaffolds. J Biomed Mater Res A 89: 1118-1124, 2009.

4. Triolo D, Dina G, Lorenzetti I, Malaguti M, Morana P, Del Carro U, Comi G, Messing A, Quattrini A and Previtali SC: Loss of glial fibrillary acidic protein (GFAP) impairs Schwann cell proliferation and delays nerve regeneration after damage. $\mathrm{J}$ Cell Sci 119: 3981-3993, 2006.

5. Heinen A, Beyer F, Tzekova N, Hartung HP and Küry P: Fingolimod induces the transition to a nerve regeneration promoting Schwann cell phenotype. Exp Neurol 271: 25-35, 2015.

6. Nishimoto S, Tanaka H, Okamoto M, Okada K, Murase T and Yoshikawa H: Methylcobalamin promotes the differentiation of Schwann cells and remyelination in lysophosphatidylcholine-induced demyelination of the rat sciatic nerve. Front Cell Neurosci 9: 298, 2014.
7. Zhao Z and Moghadasian MH: Chemistry, natural sources, dietary intake and pharmacokinetic properties of ferulic acid: A review. Food Chem 109: 691-702, 2008.

8. Chen XH, Lin ZZ, Liu AM, Ye JT, Luo Y, Luo YY, Mao XX, Liu PQ and Pi RB: The orally combined neuroprotective effects of sodium ferulate and borneol against transient global ischaemia in C57 BL/6J mice. J Pharm Pharmacol 62: 915-923, 2010.

9. Cheng CY, Su SY, Tang NY, Ho TY, Chiang SY and Hsieh CL: Ferulic acid provides neuroprotection against oxidative stress-related apoptosis after cerebral ischemia/reperfusion injury by inhibiting ICAM-1 mRNA expression in rats. Brain Res 1209: 136-150, 2008.

10. Yabe T, Hirahara H, Harada N, Ito N, Nagai T, Sanagi T and Yamada H: Ferulic acid induces neural progenitor cell proliferation in vitro and in vivo. Neuroscience 165: 515-524, 2010.

11. Jiang M, Zhuge X, Yang Y, Gu X and Ding F: The promotion of peripheral nerve regeneration by chitooligosaccharides in the rat nerve crush injury model. Neurosci Lett 454: 239-243, 2009.

12. Wang H, Liu S, Tian Y, Wu X, He Y, Li C, Namaka M, Kong J, Li H and Xiao L: Quetiapine inhibits microglial activation by neutralizing abnormal STIM1-mediated intercellular calcium homeostasis and promotes myelin repair in a cuprizone-induced mouse model of demyelination. Front Cell Neurosci 9: 492, 2015.

13. Fei W, Aixi Y, Danmou X, Wusheng K, Zhengren P and Ting R: The mood stabilizer valproic acid induces proliferation and myelination of rat Schwann cells. Neurosci Res 70: 383-390, 2011.

14. He B, Tao HY and Liu SQ: Neuroprotective effects of carboxymethylated chitosan on hydrogen peroxide induced apoptosis in Schwann cells. Eur J Pharmacol 740: 127-134, 2014.

15. Sasagasako N, Toda K, Hollis M and Quarles RH: Myelin gene expression in immortalized Schwann cells: relationship to cell density and proliferation. J Neurochem 66: 1432-1439, 1996.

16. Ju DT, Kuo WW, Ho TJ, Paul CR, Kuo CH, Viswanadha VP, Lin CC, Chen YS, Chang YM and Huang CY: Protocatechuic acid from alpinia oxyphylla induces schwann cell migration via ERK1/2, JNK and p38 activation. Am J Chin Med 43: 653-665, 2015.

17. Varejão AS, Cabrita AM, Meek MF, Bulas-Cruz J, Melo-Pinto P, Raimondo S, Geuna S and Giacobini-Robecchi MG: Functional and morphological assessment of a standardized rat sciatic nerve crush injury with a non-serrated clamp. J Neurotraum 21: 1652-1670, 2004.

18. Gupta R, Truong L, Bear D, Chafik D, Modafferi E and Hung CT:Shear stress alters the expression of myelin-associated glycoprotein (MAG) and myelin basic protein (MBP) in Schwann cells. J Orthop Res 23: 1232-1239, 2005.

19. Napoli I, Noon LA, Ribeiro S, Kerai AP, Parrinello S, Rosenberg LH, Collins MJ, Harrisingh MC, White IJ, Woodhoo A and Lloyd AC: A central role for the ERK-signaling pathway in controlling schwann cell plasticity and peripheral nerve regeneration in vivo. Neuron 73: 729-742, 2012.

20. Schnaar RL and Lopez PH: Myelin-associated glycoprotein and its axonal receptors. J Neurosci Res 87: 3267-3276, 2009.

21. Painter MW, Brosius Lutz A, Cheng YC, Latremoliere A, Duong K, Miller CM, Posada S, Cobos EJ, Zhang AX, et al: Diminished Schwann cell repair responses underlie age-associated impaired axonal regeneration. Neuron 83: 331-343, 2014.

22. Ishii A, Furusho M and Bansal R: Sustained activation of ERK1/2 MAPK in oligodendrocytes and schwann cells enhances myelin growth and stimulates oligodendrocyte progenitor expansion. J Neurosci 33: 175-186, 2013.

23. Newbern JM, Li XY, Shoemaker SE, Zhou J, Zhong J, Wu Y, Bonder D, Hollenback S, Coppola G, Geschwind DH, et al: Specific functions for ERK/MAPK signaling during PNS development. Neuron 69: 91-105, 2011. 